\title{
INTEGRABLE FOUR-COMPONENT SYSTEMS OF CONSERVATION LAWS AND LINEAR CONGRUENCES IN $\mathbb{P}^{5}$
}

\author{
S. I. AGAFONOV \\ Fachbereich Mathematik und Informatik, Martin-Luther-Universität Halle-Wittenberg, \\ D-06099 Halle (Saale), Germany \\ e-mail: Agafonov@mathematik.uni-halle.de \\ and E. V. FERAPONTOV \\ Department of Mathematical Sciences, Loughborough University, \\ Loughborough, Leicestershire LE11 3TU, United Kingdom \\ e-mail: E.V.Ferapontov@lboro.ac.uk
}

(Received 2 November, 2003; accepted 1 January, 2004)

\begin{abstract}
We propose a differential-geometric classification of the fourcomponent hyperbolic systems of conservation laws which satisfy the following properties: (a) they do not possess Riemann invariants; (b) they are linearly degenerate; (c) their rarefaction curves are rectilinear; (d) the cross-ratio of the four characteristic speeds is harmonic. This turns out to provide a classification of projective congruences in $\mathbb{P}^{5}$ whose developable surfaces are planar pencils of lines, each of these lines cutting the focal variety at points forming a harmonic quadruplet. Symmetry properties and the connection of these congruences to Cartan's isoparametric hypersurfaces are discussed.
\end{abstract}

2000 Mathematics Subject Classification. 53A25, 53B50, 35L65.

1. Introduction. Systems of conservation laws of Temple's class and the projective theory of congruences. Hyperbolic systems of conservation laws

$$
u_{t}^{i}=f^{i}(u)_{x}=v_{j}^{i}(u) u_{x}^{j}, \quad v_{j}^{i}=\frac{\partial f^{i}}{\partial u^{j}}, \quad i=1, \ldots, n,
$$

naturally arise in a variety of physical applications and are known to possess a rich mathematical and geometric structure [10], [11], [16], [6] and [17]. It was observed recently that many constructions in the theory of systems of conservation laws (1) are, in a sense, parallel to that of the projective theory of congruences. The correspondence proposed in [3] and [4] associates with any system (1) an $n$-parameter family of lines

$$
y^{i}=u^{i} y^{0}-f^{i}(u), \quad i=1, \ldots, n
$$

in $(n+1)$-dimensional projective space $\mathbb{P}^{n+1}$ with affine coordinates $y^{0}, \ldots, y^{n}$. In the case $n=2$ we obtain a two-parameter family, or a congruence of lines in $\mathbb{P}^{3}$. In the 19 th century the theory of congruences was one of the most popular chapters of classical differential geometry (see, e.g., [9]). We keep the name "congruence" for any $n$-parameter family of lines (2) in $\mathbb{P}^{n+1}$. 
In this section we give a brief review of the necessary material from [3], [4] and [18]. Let $\lambda^{i}(u)$ be the eigenvalues of the matrix $v_{j}^{i}(u)$, called the characteristic speeds of system (1). Assume they are real and pairwise distinct. Such systems are called strictly hyperbolic. Let $\xi_{i}=\left(\xi_{i}^{1}, \ldots, \xi_{i}^{n}\right)^{T}$ be the corresponding eigenvectors: $v \xi_{i}=\lambda^{i} \xi_{i}$, or, in components, $v_{k}^{s} \xi_{i}^{k}=\lambda^{i} \xi_{i}^{s}$. We denote by $L_{i}=\xi_{i}^{k} \frac{\partial}{\partial u^{k}}$ the Lie derivative in the direction of $\xi_{i}$. It is convenient to introduce the expansions

$$
\left[L_{i}, L_{j}\right]=c_{i j}^{k} L_{k}, \quad c_{i j}^{k}=-c_{j i}^{k} .
$$

In the theory of hydrodynamic type systems the rarefaction curves play a crucial role. Recall that rarefaction curves are integral curves of the eigenvectors $\xi_{i}$. Thus, there are $n$ families of rarefaction curves, and for any point in $u$-space there is exactly one rarefaction curve from each family passing through it. By virtue of the correspondence (2), a curve in $u$-space defines a ruled surface, i.e., a one-parameter family of lines in $\mathbb{P}^{n+1}$.

THEOREM 1. [3] Ruled surfaces defined by rarefaction curves of the $i$-th family are developable, i.e., their rectilinear generators are tangential to a curve. This curve can be parametrized in the form

$$
y^{0}=\lambda^{i}, \quad y^{1}=u^{1} \lambda^{i}-f^{1}(u), \ldots, \quad y^{n}=u^{n} \lambda^{i}-f^{n}(u),
$$

where $u$ varies along the rarefaction curve.

The curve (4) constitutes a singular locus of the developable surface which is called its cuspidal edge. The collection of all cuspidal edges corresponding to rarefaction curves of the i-th family defines the so-called focal hypersurface $M_{i} \subset \mathbb{P}^{n+1}$. In our case parametric equations of $M_{i}$ coincide with (4), where $u$ is now allowed to take all possible values. By a construction, each line of the congruence (2) is tangential to the focal hypersurface $M_{i}$. The idea of focal hypersurfaces is obviously borrowed from optics: thinking of the lines of a congruence as rays of light, one can intuitively imagine focal hypersurfaces as the locus in $\mathbb{P}^{n+1}$ where the light concentrates (this explains why in German literature focal hypersurfaces are called 'Brennflächen', which can be translated as 'burning surfaces').

Since the system of conservation laws (1) is strictly hyperbolic, there are precisely $n$ developable surfaces passing through a line of the congruence (2), and each line is tangential to $n$ focal hypersurfaces.

In the theory of weak solutions of systems (1) the shock curves play a fundamental role. The shock curve with vertex at $u_{0}$ is the set of points in the $u$-space such that

$$
\sigma\left(u^{i}-u_{0}^{i}\right)+f^{i}(u)-f^{i}\left(u_{0}\right)=0, \quad i-1, \ldots, n,
$$

for some function $\sigma\left(u, u_{0}\right)$. For any $u$ on the shock curve, the discontinuous function

$$
\begin{aligned}
& u(x, t)=u_{0}, \quad x<\sigma t, \\
& u(x, t)=u, \quad x>\sigma t,
\end{aligned}
$$

is a weak solution of (1). Shock curves, like rarefaction curves, define special ruled surfaces of the congruence (2); their geometry was clarified in [3].

Lax showed that a shock curve with vertex at a generic point $u_{0}$ splits into $n$ branches, the $i$ th branch being $C^{2}$-tangent to the associated rarefaction curve of the $i$ th family passing through $u_{0}$. 
As pointed out by a number of authors, there are situations where shock curves coincide with their associated rarefaction curves. Systems with coinciding shock and rarefaction curves were studied by Temple [18]. His main result can be formulated as follows.

THEOREM 2. [18] Rarefaction curves of the ith family coincide with the associated branches of the shock curve if and only if either

1) every rarefaction curve of the ith family is a straight line in the u-space, or

2) the characteristic speed $\lambda^{i}$ is constant along rarefaction curves of the ith family: $L_{i}\left(\lambda^{i}\right)=0$.

Systems satisfying the condition 2 are known as linearly degenerate. Both these conditions have a very natural geometric interpretation.

THEOREM 3. [3] Rarefaction curves of the ith family are straight lines if and only if the associated developable surfaces are planar, that is, their cuspidal edges are plane curves.

THEOREM 4. [3] The characteristic speed $\lambda^{i}$ is linearly degenerate if and only if the associated developable surfaces are conical, that is, their generators meet at a point. The corresponding focal hypersurface $M_{i}$ degenerates into a submanifold of codimension two.

As demonstrated in [3] and [4], theorems 3 and 4 provide an elementary geometric proof of Theorem 2 .

In what follows, we consider systems (1) which simultaneously satisfy both conditions of Theorem 2, namely, all rarefaction curves are rectilinear (in u-space), and all characteristic speeds $\lambda^{i}$ are linearly degenerate. Systems of this type naturally arise in the theory of equations of associativity of 2D topological field theory [5]. We will call them T-systems for short. In view of Theorems 3 and 4, developable surfaces of the corresponding congruence (2) are planar and conical simultaneously, and hence are planar pencils of lines. The corresponding focal hypersurfaces $M_{i}$ degenerate into $n$ submanifolds of codimension 2, and the lines of the congruence (2) intersect each of them. It may happen that the $(n-1)$-dimensional submanifold $M_{i}$ degenerates into a linear subspace of codimension 2 . This is closely related to the property for system (1) to possess Riemann invariants.

Definition. The Riemann invariant for $i$ th characteristic speed $\lambda^{i}$ is a function $R^{i}(u)$ such that

$$
R_{t}^{i}=\lambda^{i} R_{x}^{i}
$$

by virtue of (1).

There is a simple criterion for the existence of Riemann invariants in terms of the coefficients $c_{j k}^{i}$ defined by (3).

Proposition 1. The characteristic speed $\lambda^{i}$ possesses a Riemann invariant if and only if $c_{j k}^{i}=0$ for any $(j, k) \neq i$.

THEOREM 5. [1] The characteristic speed $\lambda^{i}$ of a T-system (1) possesses a Riemann invariant iff the corresponding focal submanifold $M_{i}$ is a linear subspace of codimension 2. 
Studying nondiagonalizable systems (1), (that is, systems which do not possess Riemann invariants), it is convenient to use the following exterior notation: let $l^{i}=\left(l_{1}^{i}(u), l_{2}^{i}(u), \ldots, l_{n}^{i}(u)\right)$ be left eigenvectors of the matrix $v_{j}^{i}(u)$ corresponding to the eigenvalues $\lambda^{i}(u)$, i.e. $l_{j}^{i} v_{k}^{j}=\lambda^{i} l_{k}^{i}$. With the eigenforms $\omega^{i}=l_{j}^{i} d u^{j}$ the system (1) is rewritten in the following exterior form:

$$
\omega^{i} \wedge\left(d x+\lambda^{i} d t\right)=0, \quad i=1, \ldots, n .
$$

The differentiation of $\omega^{i}$ and $\lambda^{i}$ gives the structure equations

$$
d \omega^{i}=\tilde{c}_{j k}^{i} \omega^{j} \wedge \omega^{k} \quad\left(\tilde{c}_{j k}^{i}=-\tilde{c}_{k j}^{i}\right), \quad d \lambda^{i}=\lambda_{j}^{i} \omega^{j},
$$

that contain all the necessary information about the system under study. If $\omega^{i}$ are normalized in such a way that $\omega^{i}\left(\xi_{j}\right)=\delta_{j}^{i}$ then the structure coefficients $\tilde{c}_{j k}^{i}$ are related to the structure coefficients $c_{j k}^{i}$ of (3) by $\tilde{c}_{j k}^{i}=-c_{j k}^{i}$.

Finally, we recall the necessary information about reciprocal transformations of systems of conservation laws. Let $B(u) d x+A(u) d t$ and $N(u) d x+M(u) d t$ be two conservation laws of system (1), understood as the one-forms which are closed by virtue of (1). In the new independent variables $X, T$ defined by

$$
d X=B(u) d x+A(u) d t, \quad d T=N(u) d x+M(u) d t,
$$

the system (1) takes the form

$$
u_{T}^{i}=V_{j}^{i}(u) u_{X}^{j}, \quad i=1, \ldots, n,
$$

where $V=(B v-A E)(M E-N v)^{-1}, E=i d$. The new characteristic speeds $\Lambda^{k}$ are

$$
\Lambda^{k}=\frac{\lambda^{k} B-A}{M-\lambda^{k} N} .
$$

Transformations of the type (8) are called reciprocal. Reciprocal transformations are known to preserve the linear degeneracy (see [7]). Moreover, if the original system is a T-system and both integrals (8) are linear combinations of the canonical integrals $u^{i} d x+f^{i} d t$,

$$
\begin{aligned}
& d X=\left(\alpha_{i} u^{i}+\alpha\right) d x+\left(\alpha_{i} f^{i}+\tilde{\alpha}\right) d t, \\
& d T=\left(\beta_{i} u^{i}+\beta\right) d x+\left(\beta_{i} f^{i}+\tilde{\beta}\right) d t,
\end{aligned}
$$

(here $\alpha_{i}, \alpha, \tilde{\alpha}, \beta_{i}, \beta, \tilde{\beta}$ are arbitrary constants), then the transformed system will be a T-system, too ([3]). Furthermore, affine transformations

$$
U^{i}=C_{j}^{i} u^{j}+D^{i}, \quad C_{j}^{i}=\text { const }, \quad D^{i}=\text { const }, \quad \operatorname{det} C_{j}^{i} \neq 0,
$$

obviously transform T-systems to T-systems.

THEOREM 6. [3] The transformation group generated by reciprocal transformations (11) and affine transformations (12) is isomorphic to the group of projective transformations of $\mathbb{P}^{n+1}$.

Thus, the classification of systems of conservation laws up to transformations (11) and (12) is equivalent to the classification of the corresponding congruences up to projective equivalence. Actually, this observation was the main reason for introducing the geometric correspondence discussed in this section. 


\section{Linear congruences.}

DEFINITION 1. The congruence (2) is called linear (or general linear) if its Plücker coordinates

$$
u^{i}, f^{i}, u^{i} f^{j}-u^{j} f^{i}
$$

satisfy $n$ linear equations of the form

$$
\alpha+\alpha_{i} u^{i}+\beta_{i} f^{i}+\alpha_{i j}\left(u^{i} f^{j}-u^{j} f^{i}\right)=0
$$

where $\alpha, \alpha_{i}, \beta_{i}, \alpha_{i j}$ are arbitrary constants.

Notice that equations (13), being linear in $f$, define $f^{i}$ as explicit functions of $u$.

Let $q$ be any fixed point in $P^{n+1}$. For the lines passing through $q$ we have $f^{i}=$ $u^{i} q^{0}-q^{i}$, which, upon the substitution into (13), implies a linear system for $u$. In general, this system possesses a unique solution, so that there exists a unique line of our congruence passing through $q$ (such congruences are said to be of order one). The focal variety $M$, also called the jump locus, consists of those $q$ for which the corresponding linear system is not uniquely solvable for $u$. One can readily see that $M$ has codimension at least two, and in the case it equals two, the developable surfaces are planar pencils of lines. Moreover, the intersection of any of these planes with the focal variety $M$ consists of a point and a plane curve of the order $n-1$.

THEOREM 7. [1] A congruence corresponding to a three-component T-system is necessarily linear.

Definition 2. A linear complex in $\mathbb{P}^{n+1}$ is a family of lines whose Plücker coordinates $P^{i j}$ are subject to a linear constraint $A_{j i} P^{i j}=0$, where $A_{i j}=-A_{j i}=$ const .

Recall that to a line in $\mathbb{P}^{n+1}$ passing through the points with homogeneous coordinates $X=\left(X^{0}: X^{1}: \ldots: X^{n+1}\right)$ and $Y=\left(Y^{0}: Y^{1}: \ldots: Y^{n+1}\right)$ there corresponds a point in the Grassmannian $\mathbb{G}(1, n+1)$ with Plücker coordinates $P^{i j}=X^{i} Y^{j}-$ $X^{j} Y^{i}, i, j=0, \ldots, n+1$. If one considers Plücker coordinates as an $(n+2) \times(n+2)$ skew-symmetric matrix $P$ of rank 2 , then any linear constraint can be written in the form $\operatorname{tr} A P=0$ where $A$ is a skew-symmetric matrix. A linear congruence is an intersection of $n$ linear complexes given by $n$ linear equations

$$
\operatorname{tr} A^{\alpha} P=0, \alpha=0, \ldots, n-1,
$$

where the matrices $A^{\alpha}$ are linearly independent. The focal variety of the linear congruence (14) is the determinantal variety

$$
M=\left\{X \in \mathbb{P}^{n+1}: \operatorname{rk}\left\{A_{i j}^{\alpha} X^{i}\right\}<n\right\},
$$

$\alpha=0, \ldots, n-1, i, j=0, \ldots, n+1$. The lines of the congruence are $n$-secants of the focal variety $M$.

Define the map $A(\mu)$ by

$$
\mathbb{P}^{n-1} \ni\left(\mu_{0}: \mu_{1}: \ldots \mu_{n-1}\right) \rightarrow A(\mu)=\sum_{\alpha} \mu_{\alpha} A^{\alpha}
$$


For even $n$, to each $\mu$ such that

$$
\operatorname{Pf}(A(\mu)) \equiv \sqrt{\operatorname{det} A(\mu)}=0
$$

there corresponds a line $l(\mu) \in M \in \mathbb{P}^{n+1}$ that belongs to the kernel of $A(\mu)$. Conversely, there exists a map $f: X \in M \rightarrow \mu \in V_{A}$ where $V_{A} \in \mathbb{P}^{n-1}$ is defined by (16). Hence, this map defines the structure of a $\mathbb{P}^{1}$-bundle over $V_{A}$ on the jump locus (15). In the case $n=4$ the geometry of focal varieties of linear congruences, also known as the Palatini scrolls, was investigated in [15] (see also [12], [14] and [19] for further properties of the Palatini scrolls).

This paper is concerned with four-component nondiagonalizable T-systems (1) whose characteristic velocities $\lambda^{i}$ form a harmonic quadruplet, i.e. their cross-ratio is equal to -1 :

$$
\frac{\left(\lambda^{1}-\lambda^{2}\right)\left(\lambda^{3}-\lambda^{4}\right)}{\left(\lambda^{2}-\lambda^{3}\right)\left(\lambda^{4}-\lambda^{1}\right)}=-1 .
$$

For T-systems (1), the condition (17) is very restrictive.

CONJECTURE 1. The congruence corresponding to a four-component T-system (1) with harmonic cross-ratio of characteristic speeds, is linear.

We prove this conjecture for T-systems without Riemann invariants. Moreover, we show that, up to reciprocal transformations, there are exactly two non-equivalent four-component T-systems without Riemann invariants, having harmonic cross-ratio of characteristic speeds.

REMARK. The condition (17) is easily verified: if the characteristic polynomial of (1) is

$$
a_{4}(u) \lambda^{4}+4 a_{3}(u) \lambda^{3}+6 a_{2}(u) \lambda^{2}+4 a_{1}(u) \lambda+a_{0}(u),
$$

then (17) is equivalent to

$$
\operatorname{det}\left|\begin{array}{lll}
a_{4} & a_{3} & a_{2} \\
a_{3} & a_{2} & a_{1} \\
a_{2} & a_{1} & a_{0}
\end{array}\right|=0
$$

see, e.g., [13].

3. Isoparametric hypersurfaces and linear congruences. In this section we describe an explicit geometric construction of the linear congruence corresponding to a four-component T-system without Riemann invariants and harmonic cross-ratio of characteristic speeds. It is associated with the Cartan isoparametric hypersurface $M^{4} \subset S^{5} \subset E^{6}$ which can be represented as the intersection of the unit sphere

$$
\left(U^{1}\right)^{2}+\left(U^{2}\right)^{2}+\left(U^{3}\right)^{2}+\left(U^{4}\right)^{2}+\left(U^{5}\right)^{2}+\left(U^{6}\right)^{2}=1
$$

with the zero level $P=0$ of the fourth order polynomial

$$
\begin{aligned}
P(U)= & -\left(\left(U^{1}\right)^{2}+\left(U^{2}\right)^{2}+\left(U^{3}\right)^{2}+\left(U^{4}\right)^{2}+\left(U^{5}\right)^{2}+\left(U^{6}\right)^{2}\right)^{2}+2\left(\left(U^{1}\right)^{2}+\left(U^{2}\right)^{2}\right. \\
& \left.+\left(U^{3}\right)^{2}-\left(U^{4}\right)^{2}-\left(U^{5}\right)^{2}-\left(U^{6}\right)^{2}\right)^{2}+8\left(U^{1} U^{4}+U^{2} U^{5}+U^{3} U^{6}\right)^{2} .
\end{aligned}
$$

Since $M^{4}$ is a non-singular 4-dimensional hypersurface, with each point $m \in M^{4}$ one can associate a unique great circle $S^{1}(m)$ in $S^{5}$ which is orthogonal to $M^{4}$, so that a 
4-parameter family of such circles is obtained. Each normal great circle intersects the "focal" surfaces $M_{ \pm} \subset S^{5}$, determined by the equations

$$
P(U)= \pm 1
$$

at four points forming a harmonic quadruplet on $S^{1}(M)$. The focal surfaces $M_{ \pm} \subset S^{5}$ are 3-dimensional, $M_{-}$being the Stiefel manifold

$$
|p|^{2}=|q|^{2}, \quad(p, q)=0,
$$

here $p=\left(U^{1}, U^{2}, U^{3}\right)^{T}, q=\left(U^{4}, U^{5}, U^{6}\right)^{T}$, whereas $M_{+}$is the cubic scroll

$$
p \wedge q=0 .
$$

Regarding $U^{i}, i=1, \ldots, 6$, as homogeneous coordinates in $P^{5}$, one arrives at a 4parameter family of lines $l(m) \subset \mathbb{P}^{5}$, each line being defined by the 2-dimensional plane of the great circle $S^{1}(m)$. Moreover, equations (22) and (23), without the original restriction $|p|^{2}+|q|^{2}=1$, specify two components of the focal variety $F^{3}$ of the congruence $\left\{l(m) \mid m \in M^{4}\right\}$, the cross-ratios of intersection points $\left\{l(m) \cap F^{3}\right\}$ being equal to -1 . Since the focal surface $F^{3}$ is 3 -dimensional, the corresponding system (1) is linearly degenerate. In the affine chart $U^{6} \neq 0$ with coordinates $y^{i}=U^{i} / U^{6}, i=$ $1, \ldots, 5$, this congruence can be parametrized by the parameters $a, b, c, d$ as follows:

$$
\begin{aligned}
& y^{1}=a y^{3}+c, \\
& y^{2}=b y^{3}+d, \\
& y^{4}=c y^{3}+\frac{a b d+c^{3}+c d^{2}-c b^{2}-c}{a c+b d}, \\
& y^{5}=d y^{3}+\frac{b a c+d^{3}+d c^{2}-d a^{2}-d}{a c+b d} .
\end{aligned}
$$

One can check by a direct computation that the congruence under consideration is linear. In the coordinates $X^{0}=U^{1}, X^{1}=U^{2}, X^{2}=U^{3}, X^{3}=U^{4}, X^{4}=U^{5}, X^{5}=U^{6}$ the matrices $A^{\alpha}$ have block forms,

$$
A^{0}=\left(\begin{array}{cc}
0 & E \\
-E & 0
\end{array}\right), \quad A^{i}=\left(\begin{array}{cc}
G^{i} & 0 \\
0 & G^{i}
\end{array}\right), \quad i=1,2,3,
$$

where $E=\operatorname{diag}\{1,1,1\}$, and the matrices $G^{i}$ are defined as follows:

$$
G^{1}=\left(\begin{array}{ccc}
0 & -1 & 0 \\
1 & 0 & 0 \\
0 & 0 & 0
\end{array}\right), \quad G^{2}=\left(\begin{array}{ccc}
0 & 0 & -1 \\
0 & 0 & 0 \\
1 & 0 & 0
\end{array}\right), \quad G^{3}=\left(\begin{array}{ccc}
0 & 0 & 0 \\
0 & 0 & -1 \\
0 & 1 & 0
\end{array}\right) .
$$

Since the congruence (24) is linear, the corresponding system (1) has rectilinear rarefaction curves. Its focal variety does not contain linear subspaces of codimension 2, therefore, the system does not possess Riemann invariants.

Developable surfaces of the congruence (which are planar pencils of lines) intersect the focal variety at a point, which is the vertex of the pencil, and a reducible plane cubic, which is a union of a straight line and a conic. The line and the point are conjugate with respect to the conic and lie on the same component of the focal variety.

REMARK 1. In $\mathbb{P}^{2}$, consider a pencil of lines and a cubic curve. Each line cuts the cubic at 3 points. If the vertex of the pencil and these 3 points form a harmonic quadruplet for each line of the pencil, then the cubic must necessarily be reducible (a 
union of a straight line and a conic), moreover, the line and the vertex are conjugate with respect to the conic. This fact seems to be classical, however, we are unable to provide the reader with an exact reference.

Proposition 2. The system (1) corresponding to the congruence (24) is integrable.

Proof. In the homogeneous coordinates $U^{i}$, the lines $\left\{l(m): m \in M^{4}\right\}$ of the congruence can be parametrized by $s_{1}, s_{2}$ as follows:

$$
\vec{U}=\overrightarrow{\mathbf{r}}(m) s_{1}+\overrightarrow{\mathbf{n}}(m) s_{2},
$$

where $\overrightarrow{\mathbf{r}}(m)$ is the position vector of the Cartan isoparametric hypersurface $M^{4} \subset S^{5} \subset$ $E^{6}$, and $\overrightarrow{\mathbf{n}}(m) \in T_{m} S^{5}$ is the unit normal. The reparametrization of this congruence in terms of $U^{3}, U^{6}$ shows that the corresponding system (1) is a reciprocal transform of the following system on $M^{4}$ (see [8]),

$$
\overrightarrow{\mathbf{r}}_{t}=\overrightarrow{\mathbf{n}}_{x}
$$

which has the exterior representation

$$
\begin{aligned}
\Omega^{1} \wedge\left(d x+\lambda^{1} d t\right) & =0, \\
\Omega^{2} & \wedge\left(d x+\lambda^{2} d t\right)=0 \\
\left(\Omega^{3}+\phi\right) & \wedge\left(d x+\lambda^{3} d t\right)=0 \\
\left(\Omega^{1}-\phi\right) & \wedge\left(d x+\lambda^{4} d t\right)=0
\end{aligned}
$$

here $\Omega^{i}$ satisfy the $S O(3)$ Maurer-Cartan equations, $d \phi=0$ and all $\lambda^{i}$ are constant. This system is equivalent to the system describing a resonant 4-wave interaction and, therefore, is integrable (see [8] for details).

REMARK 2. General linear congruences in $\mathbb{P}^{4}$ are obtained from the Cartan isoparametric hypersurface $M^{3} \subset S^{4} \subset E^{5}$ by the analogous construction. The classification of the corresponding T-systems is given in [1]. Systems of that type proved to be nondiagonalizable but integrable. The essential difference is that the "focal" submanifolds $M_{ \pm}$are antipodal in $S^{4}$ so that the focal variety of the congruence is irreducible.

REMARK 3. The proof of Proposition 2 implies that the system (1) corresponding to the congruence (24) has the exterior representation (28), however, $\lambda^{i}$ are no longer constant.

Introducing the potentials $u, v$ by

$$
u_{x x}=a, \quad u_{x t}=-c, \quad v_{x x}=b, \quad v_{x t}=-d,
$$

one can rewrite this hydrodynamic type system as a pair of two second order equations

$$
u_{x t}=-\operatorname{det}\left(\begin{array}{ccc}
u_{t t} & u_{x t} & v_{x t} \\
u_{x t} & u_{x x} & v_{x x} \\
v_{x x} & -v_{x t} & u_{x t}
\end{array}\right), \quad v_{x t}=-\operatorname{det}\left(\begin{array}{ccc}
v_{t t} & v_{x t} & u_{x t} \\
v_{x t} & v_{x x} & u_{x x} \\
u_{x x} & -u_{x t} & v_{x t}
\end{array}\right) \text {. }
$$

Recall that three-component T-systems can be cast into the form

$$
a_{t}=b_{x}, \quad b_{t}=c_{x}, \quad c_{t}=f(a, b, c)_{x}
$$


by an appropriate reciprocal transformation (11) combined with an affine change of dependent variables. The system (30) can be rewritten as a single third order PDE

$$
\frac{\partial^{3} u}{\partial t^{3}}=f\left(\frac{\partial^{3} u}{\partial x^{3}}, \frac{\partial^{3} u}{\partial x^{2} \partial t}, \frac{\partial^{3} u}{\partial x \partial t^{2}}\right)
$$

after the substitution $a=\frac{\partial^{3} u}{\partial x^{3}}, b=\frac{\partial^{3} u}{\partial x^{2} \partial t}, c=\frac{\partial^{3} u}{\partial x \partial t^{2}}$. According to the criterion found in [2], the analogous change of variables for the four-component system (1) corresponding to the congruence (24) does not exist.

4. Symmetry properties. Consider a linear congruence in $\mathbb{P}^{n+1}$ specified by a collection of $n$ skew-symmetric matrices $A^{\alpha}$ as in (14). For any $G \in G L(n+2, R)$, the Pfaffian of $A(\mu)$ transforms as

$$
\operatorname{Pf}\left(G^{T} A(\mu) G\right)=|\operatorname{det} G| \operatorname{Pf}(A(\mu)) .
$$

Hence, all projectively equivalent congruences have the same variety $V_{A} \subset \mathbb{P}^{n-1}$ defined by (16). Notice that the matrices $A^{\alpha}$ chosen as in (25) form a basis of the Lie algebra $\operatorname{so}(3) \times \operatorname{so}(2)$ represented in $s o(6)$ :

$$
\left[A^{0}, A^{i}\right]=0, i=1,2,3, \quad\left[A^{1}, A^{2}\right]=A^{3},\left[A^{2}, A^{3}\right]=A^{1},\left[A^{3}, A^{1}\right]=A^{2} .
$$

Therefore, the corresponding Lie group $S O(3) \times S O(2) \subset S O(6)$ leaves the congruence under consideration invariant:

$$
G^{T} A(\mu) G^{T}=\mu_{\alpha} G^{-1} A^{\alpha} G=\mu_{\alpha} C_{\beta}^{\alpha} A^{\beta}=\tilde{\mu}_{\beta} A^{\beta}=A(\tilde{\mu}),
$$

where $C_{\beta}^{\alpha}$ is the adjoint representation of $S O(3) \times S O(2)$, and

$$
\tilde{\mu}_{\beta}=\mu_{\alpha} C_{\beta}^{\alpha} .
$$

On the other hand,

$$
\operatorname{Pf}\left(G^{T} A(\mu) G\right)=|\operatorname{det} G| \operatorname{Pf}(A(\mu))=\operatorname{Pf}(A(\mu))=\operatorname{Pf}(A(\tilde{\mu})),
$$

so that (32) gives a symmetry of $V_{A}$. Since the kernel of the adjoint representation is $S O(2)$, this symmetry group is $S O(3)$. The Pfaffian of the congruence is factorized as

$$
\operatorname{Pf}(A(\mu))=\mu_{0}\left(\mu_{0}^{2}-\mu_{1}^{2}-\mu_{2}^{2}-\mu_{3}^{2}\right)
$$

so that the cubic surface $V_{A}$ degenerates into a union of the plane

$$
\mu_{0}=0
$$

and the quadric

$$
\mu_{1}^{2}+\mu_{2}^{2}+\mu_{3}^{2}=\mu_{0}^{2}
$$

The cubic scroll (23) and the intersection of quadrics (22) are $\mathbb{P}^{1}$-bundles over the plane (33) and the quadric (34), respectively. Considering (33) as an infinite plane in $\mathbb{P}^{3}$, one can look at transformations (32) as the $S O(3)$ symmetry of the quadric (34). 
REMARK 1 . The proposed interpretation of the $S O(3) \times S O(2)$ symmetry of the congruence as the orthogonal group, is only valid under a special choice of the basis (25). The general projective transformation destroys it, but retains the symmetry group. Therefore, any congruence projectively equivalent to (24) has a symmetry group isomorphic to $S O(3) \times S O(2)$. In terms of the system (1), this symmetry is interpreted as an autoreciprocal transformation, i.e., as a reciprocal transformation which, after being combined with a local change of field variables, leaves the equation (1) invariant.

REMARK 2. This symmetry can also be read off the focal varieties (23) and (22). Indeed, if one represents the coordinates $U$ in the form of a $3 \times 2$ matrix $r=(p, q)$, then (23) and (22) become manifestly invariant under the linear transformations

$$
r \rightarrow g_{1} r g_{2}, \quad g_{1} \in S O(3), \quad g_{2} \in S O(2)
$$

Here $g_{1}$ is a simultaneous rotation of $p$ and $q, A^{i}$ being infinitesimal generators of such rotations. The transformation $g_{2}$ can be interpreted as a rotation in the 2-dimensional plane spanned by $p$ and $q$, represented by the matrix $A^{0}$ in the basis (25).

5. Isoparametric hypersurfaces in a pseudoeuclidean space and linear congruences. The congruence (24) has a pseudoeuclidean counterpart. One can start with the same focal varieties (22) and (23), where now $(p, q)$ is a scalar product of the signature $(2,1)$ defined by the matrix

$$
H=\left(\begin{array}{lll}
0 & 0 & 1 \\
0 & 1 & 0 \\
1 & 0 & 0
\end{array}\right),
$$

and consider a linear congruence formed by its four-secants. The basis of linear constraints $A^{\alpha}$ can be chosen as

$$
A^{0}=\left(\begin{array}{cc}
0 & H \\
-H & 0
\end{array}\right)
$$

and $A^{1}, A^{2}, A^{3}$ as in (25). For this congruence the Pfaffian reads:

$$
\operatorname{Pf}(A(\mu))=\mu_{0}\left(\mu_{0}^{2}+2 \mu_{1} \mu_{3}+\mu_{2}^{2}\right) .
$$

In the affine chart $U^{2} \neq 0$ with coordinates $y^{i}=U^{i} / U^{2}, i=1,3,4,5,6$, this congruence is parametrized by $a, b, c, d$ as follows:

$$
\begin{aligned}
& y^{1}=a y^{5}+\frac{2 a^{2} b-a c d-a+b c^{2}}{a d+b c}, \\
& y^{3}=b y^{5}+\frac{2 a b^{2}-b c d-b+a d^{2}}{a d+b c}, \\
& y^{4}=c y^{5}+a, \\
& y^{5}=d y^{5}+b .
\end{aligned}
$$

REMARK. This congruence has a symmetry group isomorphic to $S O(2,1) \times S O(2)$, which is also the symmetry group of the focal variety. This symmetry is interpreted 
similarly to (35), with the only difference that $g_{1} \in S O(2,1)$, whereas $g_{2} \in S O(2)$ leaves (22) invariant regardless of the signature.

With the potentials $u, v$ defined by

$$
u_{x x}=-c, u_{x t}=a, v_{x x}=-d, v_{x t}=b,
$$

the corresponding system (1) takes the form:

$$
u_{x t}=\operatorname{det}\left(\begin{array}{ccc}
u_{t t} & u_{x t} & v_{x t} \\
u_{x t} & u_{x x} & v_{x x} \\
-u_{x x} & u_{x t} & -v_{x t}
\end{array}\right), \quad v_{x t}=\operatorname{det}\left(\begin{array}{ccc}
v_{t t} & v_{x t} & u_{x t} \\
v_{x t} & v_{x x} & u_{x x} \\
-v_{x x} & v_{x t} & -u_{x t}
\end{array}\right) \text {. }
$$

6. The classification result. In this short final section we formulate the main result of classification of four-component non-diagonalizable T-systems having harmonic cross-ratio of characteristic speeds. This result is differential-geometric, as we do not assume the linearity of the corresponding congruence.

THEOREM 8. Let a congruence in $\mathbb{P}^{5}$ have the following properties:

- its Plücker image in $\mathbb{G}(1,5)$ is connected,

- its focal variety has codimension 2 and does not contain linear subspaces of codimension 2,

- its developable surfaces are planar pencils of lines, transversal to the focal varieties,

- each line cuts the focal surface at four points forming a harmonic quadruplet, each pair of these points not coinciding identically.

Then this congruence is projectively equivalent over the reals to either (24) or (37).

The proof of this theorem is purely technical and given in the Appendix.

7. Appendix. Proof of Theorem 8. The most difficult part of the theorem is the following lemma.

LEMMA 1. If (1) is the system corresponding to a congruence with the properties as in Theorem 8 , then its eigenforms can be normalized so that the exterior representation takes the form (28) where $\Omega^{i}$ satisfy the Maurer-Cartan equations of the $\operatorname{SO}(3)$ or $\operatorname{SO}(2,1)$ groups.

The proof requires a long computation bringing a certain exterior differential system into involutive form. To derive and analyze these equations we used computer algebra system MAPLE 7. We do not present all intermediate formulas as they are extremely awkward. Let us only sketch the proof and final results.

Since the system under study does not possess Riemann invariants, at least one of the coefficients $\tilde{c}_{j k}^{i}$ with $j, k \neq i$ in (7) does not vanish for each $i=1, \ldots, 4$. By an appropriate normalization $\omega^{i} \rightarrow r^{i} \omega^{i}, r^{i} \neq 0$, one can make them constant. (We allow $r^{i}$ to be complex to treat all cases on equal footing). Thus, there are $3^{4}=81$ possibilities to consider. As the equation (17) is invariant under the permutations $\{(1,2,3,4),(2,1,3,4),(1,2,4,3),(2,1,4,3),(3,4,1,2),(4,3,2,1)$, $(4,3,1,2),(3,4,2,1)\}$, only 15 of these possibilities are essentially different. They are presented in the following table, where $i, j, k$ are the indices of non-zero $\tilde{c}_{j k}^{i}$. 


\begin{tabular}{|c|c|c|c|c|c|c|c|c|c|c|c|c|c|c|c|}
\hline \multicolumn{11}{|c|}{ Different cases } \\
\hline & 1 & 2 & 3 & 4 & 5 & 6 & 7 & 8 & 9 & 10 & 11 & 12 & 13 & 14 & 15 \\
\hline $\mathrm{i}$ & $\mathrm{j}, \mathrm{k}$ & $\mathrm{j}, \mathrm{k}$ & $\mathrm{j}, \mathrm{k}$ & $\mathrm{j}, \mathrm{k}$ & $\mathrm{j}, \mathrm{k}$ & $\mathrm{j}, \mathrm{k}$ & $\mathrm{j}, \mathrm{k}$ & $\mathrm{j}, \mathrm{k}$ & $\mathrm{j}, \mathrm{k}$ & $\mathrm{j}, \mathrm{k}$ & $\mathrm{j}, \mathrm{k}$ & $\mathrm{j}, \mathrm{k}$ & $\mathrm{j}, \mathrm{k}$ & $\mathrm{j}, \mathrm{k}$ & $\mathrm{j}, \mathrm{k}$ \\
\hline 1 & 2,3 & 2,3 & 3,4 & 3,4 & 3,4 & 2,3 & 2,3 & 3,4 & 2,3 & 2,3 & 2,3 & 2,3 & 2,3 & 3,4 & 2,3 \\
\hline 2 & 4,1 & 1,3 & 3,1 & 3,4 & 3,1 & 1,3 & 4,1 & 3,1 & 4,1 & 1,3 & 1,3 & 4,1 & 4,1 & 3,1 & 4,1 \\
\hline 3 & 1,2 & 1,2 & 1,2 & 1,2 & 1,2 & 1,2 & 1,2 & 1,2 & 1,2 & 4,1 & 4,1 & 4,1 & 4,1 & 2,4 & 2,4 \\
\hline 4 & 1,2 & 1,2 & 1,2 & 1,2 & 3,1 & 3,1 & 3,1 & 3,2 & 3,2 & 1,2 & 1,3 & 1,3 & 2,3 & 1,2 & 3,1 \\
\hline
\end{tabular}

The eigenforms $\omega^{i}$ constitute a basis, therefore, the differential of any function $u$ can be decomposed as $d u=u_{i} \omega^{i}$ where, for brevity, we use the notation $u_{i}=L_{i}(u)$. Let $\vec{u}=\left(u^{1}, u^{2}, u^{3}, u^{4}\right)$ be the densities of conservation laws for which rarefaction curves are linear. Then there exist scalars $p_{i}$ such that

$$
\overrightarrow{u_{i i}}=p_{i} \overrightarrow{u_{i}}
$$

Differentiating the relations $d u^{j}=u_{i}^{j} \omega^{i}, d f^{j}=\lambda^{i} u_{i}^{j} \omega^{i}$ and equating to zero coefficients of $\omega^{i} \wedge \omega^{j}$, one obtains all mixed second derivatives $u_{i k}^{j}, i \neq k$. Thus, one has

$$
d \overrightarrow{u_{i}}=\vec{U}_{i j}\left(\lambda^{k}, \lambda_{l}^{k}, p_{n}, \tilde{c}_{q r}^{s}, \vec{u}_{m}\right) \omega^{j}
$$

Note that $\vec{U}_{i j}$ are linear in $\vec{u}_{m}$. Differentiating these equations and equating to zero coefficients of $\omega^{i} \wedge \omega^{j}$, one obtains $4 \times C_{4}^{2}=24$ equations which are linear with respect to $\vec{u}_{j}$. Since $u^{i}$ are functionally independent, these equations split with respect to $\vec{u}_{j}$. As a result, one gets $24 \times 4=96$ differential equations for $\lambda^{k}, p_{i}$ and the structure coefficients $\tilde{c}_{q r}^{s}$. The analysis of these equations along with (17), the linear degeneracy conditions

$$
\lambda_{i}^{i}=0, \quad i=1, \ldots, 4
$$

and the equations $d\left(d\left(\omega^{i}\right)\right)=0, d\left(d\left(\lambda^{i}\right)\right)=0$, shows that the structure equations for the system under study are

$$
\begin{aligned}
& d \omega^{1}=\frac{1}{2} \omega^{2} \wedge \omega^{3}+\frac{1}{2} \omega^{2} \wedge \omega^{4}, \quad d \omega^{2}=\frac{1}{2} \omega^{3} \wedge \omega^{1}+\frac{1}{2} \omega^{4} \wedge \omega^{1}, \\
& d \omega^{3}=\omega^{1} \wedge \omega^{2}, \quad d \omega^{4}=\omega^{1} \wedge \omega^{2} .
\end{aligned}
$$

These equations imply that $\Omega^{1}=\omega^{1}, \Omega^{2}=\omega^{2}, \Omega^{3}=\left(\omega^{3}+\omega^{4}\right) / 2$ satisfy the structure equations of the $S O(3)$-group, and $d \phi=d\left(\omega^{3}-\omega^{4}\right) / 2=0$. The complex normalization

$$
\omega^{1}=i \Omega^{1}, \quad \omega^{2}=\Omega^{2}, \quad \omega^{3}=i \Omega^{3}+\phi, \quad \omega^{4}=i \Omega^{4}-\phi
$$

gives the structure equations

$$
\begin{array}{cl}
d \omega^{1}=\frac{1}{2} \omega^{2} \wedge \omega^{3}+\frac{1}{2} \omega^{2} \wedge \omega^{4}, & d \omega^{2}=-\frac{1}{2} \omega^{3} \wedge \omega^{1}-\frac{1}{2} \omega^{4} \wedge \omega^{1}, \\
d \omega^{3}=\omega^{1} \wedge \omega^{2}, & d \omega^{4}=\omega^{1} \wedge \omega^{2}
\end{array}
$$

for the pseudo-Euclidean counterpart. Q.E.D. 
REMARK. Given the structure equations (41), one can compute $p_{i}$,

$$
\begin{aligned}
& p_{1}=\frac{2 \lambda_{1}^{2}\left(\lambda^{3}-\lambda^{1}\right)}{\left(\lambda^{2}-\lambda^{1}\right)\left(\lambda^{2}-\lambda^{3}\right)}+\frac{2 \lambda_{1}^{3}\left(\lambda^{2}-\lambda^{1}\right)}{\left(\lambda^{3}-\lambda^{1}\right)\left(\lambda^{3}-\lambda^{2}\right)}, \\
& p_{2}=\frac{2 \lambda_{2}^{1}\left(\lambda^{3}-\lambda^{2}\right)}{\left(\lambda^{1}-\lambda^{2}\right)\left(\lambda^{1}-\lambda^{3}\right)}+\frac{2 \lambda_{2}^{3}\left(\lambda^{1}-\lambda^{2}\right)}{\left(\lambda^{3}-\lambda^{1}\right)\left(\lambda^{3}-\lambda^{2}\right)}, \\
& p_{3}=\frac{2 \lambda_{3}^{1}\left(\lambda^{2}-\lambda^{3}\right)}{\left(\lambda^{1}-\lambda^{2}\right)\left(\lambda^{1}-\lambda^{3}\right)}+\frac{2 \lambda_{3}^{2}\left(\lambda^{1}-\lambda^{3}\right)}{\left(\lambda^{2}-\lambda^{1}\right)\left(\lambda^{2}-\lambda^{3}\right)}, \\
& p_{4}=\frac{2 \lambda_{4}^{2}\left(\lambda^{1}-\lambda^{3}\right)}{\left(\lambda^{2}-\lambda^{3}\right)\left(\lambda^{1}-\lambda^{2}\right)}+\frac{2 \lambda_{4}^{1}\left(\lambda^{2}-\lambda^{3}\right)}{\left(\lambda^{1}-\lambda^{2}\right)\left(\lambda^{3}-\lambda^{1}\right)},
\end{aligned}
$$

as well as all second derivatives of $\lambda^{1}, \lambda^{2}$ and $\lambda^{3}$, as functions $L_{j k}^{i}\left(\lambda^{m}, \lambda_{l}^{n}\right)$ of $\lambda^{1}, \lambda^{2}$, $\lambda^{3}$ and first derivatives thereof. These satisfy the above mentioned 96 equations along with $d\left(d\left(\lambda^{i}\right)\right)=0$, equations (40), (17) and their differentials. Moreover, the system

$$
\begin{aligned}
& d \lambda^{1}=\lambda_{2}^{1} \omega^{2}+\lambda_{3}^{1} \omega^{3}+\lambda_{4}^{1} \omega^{4} \\
& d \lambda^{2}=\lambda_{1}^{2} \omega^{1}+\lambda_{3}^{2} \omega^{3}+\lambda_{4}^{2} \omega^{4} \\
& d \lambda^{3}=\lambda_{1}^{3} \omega^{1}+\lambda_{2}^{3} \omega^{2}+\left(2 \lambda_{4}^{2} \frac{\left(\lambda^{3}-\lambda^{1}\right)^{2}}{\left(\lambda^{2}-\lambda^{1}\right)^{2}}+2 \lambda_{4}^{1} \frac{\left(\lambda^{2}-\lambda^{3}\right)^{2}}{\left(\lambda^{2}-\lambda^{1}\right)^{2}}\right) \omega^{4},
\end{aligned}
$$

for $\lambda^{1}, \lambda^{2}, \lambda^{3}$ (here $\lambda_{4}^{3}$ is found from (17) and $\lambda_{4}^{4}=0$ ) along with the equations

$$
d \lambda_{j}^{i}=L_{j k}^{i}\left(\lambda^{m}, \lambda_{l}^{n}\right) \omega^{k}
$$

for the 8 first derivatives $\lambda_{2}^{1}, \lambda_{3}^{1}, \lambda_{4}^{1}, \lambda_{1}^{2}, \lambda_{3}^{2}, \lambda_{4}^{2}, \lambda_{1}^{3}, \lambda_{2}^{3}$, turns out to be in involution. For the case $S O(3) \times S O(2)$ the exact formulas for $L_{j k}^{i}$ are given below.

Proof of Theorem 8: Let $\lambda^{i}$ and $\omega^{i}$ be characteristic speeds and the eigenforms of the system corresponding to (24). Then there exist 4 conservation laws with functionally independent densities $u^{i}$ rectifying rarefaction curves. One can consider $u^{i}$ as local coordinates parametrizing the congruence. Let $\tilde{\lambda}^{i}$ and $\tilde{\omega}^{i}$ be the characteristic speeds and the eigenforms of the system corresponding to a congruence $G$ which satisfies the hypothesis of the theorem. According to lemma 1, the structure equations for $\tilde{\omega}^{i}$ are either as for (24) or (37). Suppose they are as for (24). (The proof for the case (37) is the same). Then one can take $\tilde{\omega}=\omega$ and consider $u^{i}$ as local coordinates for the congruence $G$ as well. Then $\lambda^{i}$ and $\tilde{\lambda}^{i}$ satisfy the same system (43), (44). We show that there exist such constants $\alpha_{i}, \alpha, \tilde{\alpha}, \beta_{i}, \beta, \tilde{\beta}$ that, locally,

$$
\tilde{\lambda}^{k}=\Lambda^{k} \equiv \frac{\lambda^{k}\left(\alpha_{i} u^{i}+\alpha\right)-\left(\alpha_{i} f^{i}+\tilde{\alpha}\right)}{\left(\beta_{i} f^{i}+\tilde{\beta}\right)-\lambda^{k}\left(\beta_{i} u^{i}+\beta\right)} \equiv \frac{\lambda^{k} B-A}{M-\lambda^{k} N}, \quad i=1,2,3 .
$$

To this end it suffices to find the constants which satisfy (45) and $\tilde{\lambda}_{j}^{i}=\Lambda_{j}^{i}$ only at one point $\vec{u}_{0}$ since the system (43), (44) is completely integrable. Let us fix some $\vec{u}_{0}$. Then (45) defines $A, B, M, N$ at $\vec{u}_{0}$ up to a common factor. Moreover, $B M-A N \neq 0$ since $\lambda^{i}$ are distinct (as well as $\tilde{\lambda}^{i}$ ). A direct computation yields

$$
\Lambda_{2}^{1}=\lambda_{2}^{1} \frac{(B M-A N)}{\left(M-\lambda^{1} N\right)^{2}}+\frac{\left(\lambda^{1}-\lambda^{2}\right)}{\left(M-\lambda^{1} N\right)^{2}}\left\{B_{2}\left(M-\lambda^{1} N\right)+N_{2}\left(\lambda^{1} B-A\right)\right\},
$$


where we have used $f_{i}=\lambda^{i} u_{i}$. Similarly,

$$
\Lambda_{2}^{3}=\lambda_{2}^{3} \frac{(B M-A N)}{\left(M-\lambda^{3} N\right)^{2}}+\frac{\left(\lambda^{3}-\lambda^{2}\right)}{\left(M-\lambda^{3} N\right)^{2}}\left\{B_{2}\left(M-\lambda^{3} N\right)+N_{2}\left(\lambda^{3} B-A\right)\right\} .
$$

The linear system

$$
\tilde{\lambda}_{2}^{1}=\Lambda_{2}^{1}, \quad \tilde{\lambda}_{2}^{3}=\Lambda_{2}^{3}
$$

defines $B_{2}, N_{2}$ uniquely since the determinant of this system is $\frac{\left(\lambda^{1}-\lambda^{2}\right)\left(\lambda^{3}-\lambda^{2}\right)\left(\lambda^{1}-\lambda^{3}\right)(A N-M B)}{\left(M-\lambda^{1} N\right)^{2}\left(M-\lambda^{3} N\right)^{2}} \neq 0$. Similarly, the equations

$$
\tilde{\lambda}_{1}^{2}=\Lambda_{1}^{2}, \quad \tilde{\lambda}_{1}^{3}=\Lambda_{1}^{3}
$$

give $B_{1}, N_{1}$. Finally, $B_{3}, N_{3}$ and $B_{4}, N_{4}$ are determined from

$$
\tilde{\lambda}_{3}^{1}=\Lambda_{3}^{1}, \quad \tilde{\lambda}_{3}^{2}=\Lambda_{3}^{2}
$$

and

$$
\tilde{\lambda}_{4}^{1}=\Lambda_{4}^{1}, \quad \tilde{\lambda}_{4}^{2}=\Lambda_{4}^{2}
$$

respectively. The $A, B, N, M$ and $B_{i}, N_{i}$ thus obtained define $\alpha_{i}, \alpha, \tilde{\alpha}, \beta_{i}, \beta, \tilde{\beta}$, which means that the system corresponding to $G$ is a reciprocal transform of the system corresponding to (24). Thus, we have also proved that $u^{i}$ are not just local coordinates for $G$, but also the "rectifying" densities of conservation laws of the corresponding system (1). Since congruences under consideration define connected manifolds in $\mathbb{G}(1,5)$, this local equivalence is extended globally. Q.E.D.

Finally, we present formulas for $L_{j k}^{i}\left(\lambda^{m}, \lambda_{l}^{n}\right)$ of (44). As $\lambda^{4}$ is found from (17) and $\lambda_{4}^{3}$ is obtained from $\lambda_{4}^{4}=0$, the permutation symmetry used in lemma 1 is lost. Therefore, we give all necessary expressions without alluding to index permutations.

$$
\begin{aligned}
\lambda_{21}^{1}= & -\lambda_{3}^{1}-\lambda_{4}^{1}, \quad \lambda_{22}^{1}=\frac{2 \lambda_{2}^{1} \lambda_{2}^{3}\left(\lambda^{1}-\lambda^{2}\right)}{\left(\lambda^{2}-\lambda^{3}\right)\left(\lambda^{1}-\lambda^{3}\right)}+\frac{2\left(\lambda_{2}^{1}\right)^{2}}{\lambda^{1}-\lambda^{3}}, \quad \lambda_{31}^{1}=\frac{1}{2} \lambda_{2}^{1}, \\
& \lambda_{32}^{1}=\lambda_{23}^{1}, \quad \lambda_{41}^{1}=\frac{1}{2} \lambda_{2}^{1}, \\
\lambda_{23}^{1}= & \frac{\left(2 \lambda^{1}-\lambda^{2}-\lambda^{3}\right) \lambda_{2}^{1} \lambda_{3}^{1}}{\left(\lambda^{3}-\lambda^{1}\right)\left(\lambda^{2}-\lambda^{1}\right)}+\frac{\left(\lambda^{1}-\lambda^{3}\right) \lambda_{2}^{1} \lambda_{3}^{2}}{\left(\lambda^{2}-\lambda^{1}\right)\left(\lambda^{2}-\lambda^{3}\right)}+\frac{\left(\lambda^{2}-\lambda^{1}\right) \lambda_{3}^{1} \lambda_{2}^{3}}{\left(\lambda^{2}-\lambda^{3}\right)\left(\lambda^{3}-\lambda^{1}\right)} \\
& -\frac{\left(\lambda^{1}-\lambda^{3}\right)^{2} \lambda_{1}^{2}}{2\left(\lambda^{2}-\lambda^{3}\right)^{2}}+\frac{\left(\lambda^{1}-\lambda^{2}\right)^{2} \lambda_{1}^{3}}{2\left(\lambda^{2}-\lambda^{3}\right)^{2}}, \\
\lambda_{24}^{1}= & \frac{\left(2 \lambda^{1}-\lambda^{2}-\lambda^{3}\right) \lambda_{2}^{1} \lambda_{4}^{1}}{\left(\lambda^{3}-\lambda^{1}\right)\left(\lambda^{2}-\lambda^{1}\right)}+\frac{\left(\lambda^{3}-\lambda^{1}\right) \lambda_{2}^{1} \lambda_{4}^{2}}{\left(\lambda^{2}-\lambda^{1}\right)\left(\lambda^{2}-\lambda^{3}\right)}+\frac{\left(\lambda^{2}-\lambda^{1}\right) \lambda_{4}^{1} \lambda_{2}^{3}}{\left(\lambda^{2}-\lambda^{3}\right)\left(\lambda^{3}-\lambda^{1}\right)} \\
& +\frac{\left(\lambda^{1}-\lambda^{3}\right)^{2} \lambda_{1}^{2}}{2\left(\lambda^{2}-\lambda^{3}\right)^{2}}-\frac{\left(\lambda^{1}-\lambda^{2}\right)^{2} \lambda_{1}^{3}}{2\left(\lambda^{2}-\lambda^{3}\right)^{2}}, \\
\lambda_{33}^{1}= & \frac{2\left(\lambda_{3}^{1}\right)^{2}}{\lambda^{1}-\lambda^{2}}+\frac{2\left(\lambda^{1}-\lambda^{3}\right) \lambda_{3}^{1} \lambda_{3}^{2}}{\left(\lambda^{2}-\lambda^{1}\right)\left(\lambda^{2}-\lambda^{3}\right)}+\frac{\left(\lambda^{3}-\lambda^{1}\right)\left(\lambda^{2}-\lambda^{1}\right)}{2\left(\lambda^{2}-\lambda^{3}\right)}, \\
& \lambda_{42}^{1}=\lambda_{24}^{1}, \quad \lambda_{43}^{1}=\lambda_{34}^{1}, \quad \lambda_{12}^{2}=\lambda_{3}^{2}+\lambda_{4}^{2},
\end{aligned}
$$




$$
\begin{aligned}
& \lambda_{34}^{1}=\frac{\left(\lambda^{1}-\lambda^{3}\right) \lambda_{4}^{1} \lambda_{3}^{2}}{\left(\lambda^{2}-\lambda^{1}\right)\left(\lambda^{2}-\lambda^{3}\right)}+\frac{\left(\lambda^{3}-\lambda^{1}\right) \lambda_{3}^{1} \lambda_{4}^{2}}{\left(\lambda^{2}-\lambda^{1}\right)\left(\lambda^{2}-\lambda^{3}\right)}+\frac{2\left(\lambda^{3}-\lambda^{2}\right) \lambda_{3}^{1} \lambda_{4}^{1}}{\left(\lambda^{2}-\lambda^{1}\right)\left(\lambda^{2}-\lambda^{3}\right)}, \\
& \lambda_{13}^{2}=\lambda_{31}^{2}, \quad \lambda_{32}^{2}=-\frac{1}{2} \lambda_{1}^{2}, \\
& \lambda_{44}^{1}=\frac{2\left(\lambda^{3}-\lambda^{2}\right)\left(\lambda_{4}^{1}\right)^{2}}{\left(\lambda^{2}-\lambda^{1}\right)\left(\lambda^{2}-\lambda^{3}\right)}+\frac{2\left(\lambda^{3}-\lambda^{1}\right) \lambda_{4}^{1} \lambda_{4}^{2}}{\left(\lambda^{2}-\lambda^{1}\right)\left(\lambda^{2}-\lambda^{3}\right)}+\frac{\left(\lambda^{3}-\lambda^{1}\right)\left(\lambda^{2}-\lambda^{1}\right)}{2\left(\lambda^{3}-\lambda^{2}\right)}, \\
& \lambda_{41}^{2}=\lambda_{14}^{2}, \quad \lambda_{42}^{2}=-\frac{1}{2} \lambda_{1}^{2}, \\
& \lambda_{11}^{2}=\frac{2\left(\lambda_{1}^{2}\right)^{2}}{\lambda^{2}-\lambda^{3}}+\frac{2\left(\lambda^{1}-\lambda^{2}\right) \lambda_{1}^{2} \lambda_{1}^{3}}{\left(\lambda^{3}-\lambda^{1}\right)\left(\lambda^{2}-\lambda^{3}\right)}, \\
& \lambda_{33}^{2}=\frac{2\left(\lambda^{3}-\lambda^{1}\right)\left(\lambda_{3}^{2}\right)^{2}}{\left(\lambda^{1}-\lambda^{2}\right)\left(\lambda^{1}-\lambda^{3}\right)}+\frac{2\left(\lambda^{2}-\lambda^{3}\right) \lambda_{3}^{1} \lambda_{3}^{2}}{\left(\lambda^{1}-\lambda^{2}\right)\left(\lambda^{1}-\lambda^{3}\right)}+\frac{\left(\lambda^{2}-\lambda^{1}\right)\left(\lambda^{2}-\lambda^{3}\right)}{2\left(\lambda^{1}-\lambda^{3}\right)}, \\
& \lambda_{14}^{2}=\frac{\left(2 \lambda^{2}-\lambda^{1}-\lambda^{3}\right) \lambda_{1}^{2} \lambda_{4}^{2}}{\left(\lambda^{2}-\lambda^{1}\right)\left(\lambda^{2}-\lambda^{3}\right)}+\frac{\left(\lambda^{3}-\lambda^{2}\right) \lambda_{4}^{1} \lambda_{1}^{2}}{\left(\lambda^{3}-\lambda^{1}\right)\left(\lambda^{2}-\lambda^{1}\right)}+\frac{\left(\lambda^{2}-\lambda^{1}\right) \lambda_{4}^{2} \lambda_{1}^{3}}{\left(\lambda^{3}-\lambda^{2}\right)\left(\lambda^{3}-\lambda^{1}\right)} \\
& -\frac{\left(\lambda^{2}-\lambda^{3}\right)^{2} \lambda_{2}^{1}}{2\left(\lambda^{3}-\lambda^{1}\right)^{2}}+\frac{\left(\lambda^{1}-\lambda^{2}\right)^{2} \lambda_{2}^{3}}{2\left(\lambda^{1}-\lambda^{3}\right)^{2}} \\
& \lambda_{31}^{2}=\frac{\left(2 \lambda^{2}-\lambda^{1}-\lambda^{3}\right) \lambda_{1}^{2} \lambda_{3}^{2}}{\left(\lambda^{2}-\lambda^{1}\right)\left(\lambda^{2}-\lambda^{3}\right)}+\frac{\left(\lambda^{2}-\lambda^{3}\right) \lambda_{3}^{1} \lambda_{1}^{2}}{\left(\lambda^{3}-\lambda^{1}\right)\left(\lambda^{2}-\lambda^{1}\right)}+\frac{\left(\lambda^{2}-\lambda^{1}\right) \lambda_{3}^{2} \lambda_{1}^{3}}{\left(\lambda^{3}-\lambda^{2}\right)\left(\lambda^{3}-\lambda^{1}\right)} \\
& -\frac{\left(\lambda^{2}-\lambda^{1}\right)^{2} \lambda_{2}^{3}}{2\left(\lambda^{3}-\lambda^{1}\right)^{2}}+\frac{\left(\lambda^{2}-\lambda^{3}\right)^{2} \lambda_{2}^{1}}{2\left(\lambda^{1}-\lambda^{3}\right)^{2}} \\
& \lambda_{34}^{2}=\frac{2\left(\lambda^{3}-\lambda^{1}\right) \lambda_{3}^{2} \lambda_{4}^{2}}{\left(\lambda^{3}-\lambda^{1}\right)\left(\lambda^{2}-\lambda^{1}\right)}+\frac{\left(\lambda^{3}-\lambda^{2}\right) \lambda_{3}^{2} \lambda_{4}^{1}}{\left(\lambda^{3}-\lambda^{1}\right)\left(\lambda^{2}-\lambda^{1}\right)}+\frac{\left(\lambda^{2}-\lambda^{3}\right) \lambda_{3}^{1} \lambda_{4}^{2}}{\left(\lambda^{3}-\lambda^{1}\right)\left(\lambda^{2}-\lambda^{1}\right)}, \\
& \lambda_{43}^{2}=\lambda_{34}^{2}, \quad \lambda_{13}^{3}=-\frac{1}{2} \lambda_{2}^{3}, \quad \lambda_{23}^{3}=\frac{1}{2} \lambda_{1}^{3}, \\
& \lambda_{44}^{2}=\frac{2\left(\lambda_{4}^{2}\right)^{2}}{\lambda^{2}-\lambda^{1}}+\frac{2\left(\lambda^{3}-\lambda^{2}\right) \lambda_{4}^{1} \lambda_{4}^{2}}{\left(\lambda^{2}-\lambda^{1}\right)\left(\lambda^{3}-\lambda^{1}\right)}+\frac{\left(\lambda^{2}-\lambda^{1}\right)\left(\lambda^{2}-\lambda^{3}\right)}{2\left(\lambda^{3}-\lambda^{1}\right)}, \\
& \lambda_{21}^{3}=\lambda_{12}^{3}-2\left(\frac{\left(\lambda^{3}-\lambda^{1}\right)^{2} \lambda_{4}^{2}}{\left(\lambda^{2}-\lambda^{1}\right)^{2}}+\frac{\left(\lambda^{2}-\lambda^{3}\right)^{2} \lambda_{4}^{1}}{\left(\lambda^{2}-\lambda^{1}\right)^{2}}\right) \text {, } \\
& \lambda_{11}^{3}=\frac{2\left(\lambda_{1}^{3}\right)^{2}}{\lambda^{3}-\lambda^{2}}+\frac{2\left(\lambda^{3}-\lambda^{1}\right) \lambda_{1}^{2} \lambda_{1}^{3}}{\left(\lambda^{2}-\lambda^{1}\right)\left(\lambda^{2}-\lambda^{3}\right)}+\frac{\left(\lambda^{3}-\lambda^{1}\right)\left(\lambda^{3}-\lambda^{2}\right)}{\lambda^{2}-\lambda^{1}}, \quad \lambda_{22}^{3}=\frac{2\left(\lambda_{2}^{3}\right)^{2}}{\lambda^{3}-\lambda^{1}} \\
& +\frac{2\left(\lambda^{3}-\lambda^{2}\right) \lambda_{2}^{1} \lambda_{2}^{3}}{\left(\lambda^{2}-\lambda^{1}\right)\left(\lambda^{3}-\lambda^{1}\right)}+\frac{\left(\lambda^{3}-\lambda^{1}\right)\left(\lambda^{2}-\lambda^{3}\right)}{\left(\lambda^{2}-\lambda^{1}\right)} \\
& \lambda_{12}^{3}=\frac{\left(2 \lambda^{3}-\lambda^{1}-\lambda^{2}\right) \lambda_{1}^{3} \lambda_{2}^{3}}{\left(\lambda^{1}-\lambda^{3}\right)\left(\lambda^{2}-\lambda^{3}\right)}+\frac{\left(\lambda^{3}-\lambda^{2}\right) \lambda_{2}^{1} \lambda_{1}^{3}}{\left(\lambda^{3}-\lambda^{1}\right)\left(\lambda^{2}-\lambda^{1}\right)}+\frac{\left(\lambda^{3}-\lambda^{1}\right) \lambda_{2}^{3} \lambda_{1}^{2}}{\left(\lambda^{2}-\lambda^{1}\right)\left(\lambda^{2}-\lambda^{3}\right)} \\
& -\frac{\left(\lambda^{2}-\lambda^{3}\right)^{2} \lambda_{3}^{1}}{\left(\lambda^{2}-\lambda^{1}\right)^{2}}+\frac{\left(\lambda^{2}-\lambda^{3}\right)^{2} \lambda_{4}^{1}}{\left(\lambda^{1}-\lambda^{2}\right)^{2}}+\frac{\left(\lambda^{3}-\lambda^{1}\right)^{2} \lambda_{3}^{2}}{\left(\lambda^{1}-\lambda^{2}\right)^{2}}+\frac{\left(\lambda^{1}-\lambda^{3}\right)^{2} \lambda_{4}^{2}}{\left(\lambda^{1}-\lambda^{2}\right)^{2}} \\
& \lambda_{14}^{3}=\frac{2\left(2 \lambda^{3}-\lambda^{1}-\lambda^{2}\right)\left(\lambda^{1}-\lambda^{3}\right) \lambda_{1}^{3} \lambda_{4}^{2}}{\left(\lambda^{1}-\lambda^{2}\right)^{2}\left(\lambda^{2}-\lambda^{3}\right)}+\frac{2\left(\lambda^{1}-\lambda^{3}\right)\left(\lambda^{2}-\lambda^{3}\right) \lambda_{4}^{1} \lambda_{1}^{2}}{\left(\lambda^{1}-\lambda^{2}\right)^{3}}+\frac{4\left(\lambda^{3}-\lambda^{2}\right) \lambda_{4}^{1} \lambda_{1}^{3}}{\left(\lambda^{1}-\lambda^{2}\right)^{2}} \\
& +\frac{2\left(\lambda^{3}-\lambda^{1}\right)^{3} \lambda_{1}^{2} \lambda_{4}^{2}}{\left(\lambda^{2}-\lambda^{3}\right)\left(\lambda^{2}-\lambda^{1}\right)^{3}}+\frac{\lambda_{2}^{3}}{2}
\end{aligned}
$$




$$
\begin{aligned}
\lambda_{24}^{3}= & \frac{2\left(2 \lambda^{3}-\lambda^{1}-\lambda^{2}\right)\left(\lambda^{3}-\lambda^{2}\right) \lambda_{2}^{3} \lambda_{4}^{1}}{\left(\lambda^{1}-\lambda^{2}\right)^{2}\left(\lambda^{3}-\lambda^{1}\right)}+\frac{2\left(\lambda^{1}-\lambda^{3}\right)\left(\lambda^{2}-\lambda^{3}\right) \lambda_{2}^{1} \lambda_{4}^{2}}{\left(\lambda^{2}-\lambda^{1}\right)^{3}}+\frac{4\left(\lambda^{3}-\lambda^{1}\right) \lambda_{4}^{2} \lambda_{2}^{3}}{\left(\lambda^{1}-\lambda^{2}\right)^{2}} \\
& +\frac{2\left(\lambda^{3}-\lambda^{2}\right)^{3} \lambda_{2}^{1} \lambda_{4}^{1}}{\left(\lambda^{3}-\lambda^{1}\right)\left(\lambda^{2}-\lambda^{1}\right)^{3}}-\frac{\lambda_{1}^{3}}{2} .
\end{aligned}
$$

AcKnowledgements. The authors thank E. Mezzetti, D. Portelli and F. Zak for clarifying discussions. SIA thanks the Department of Mathematical Sciences at Loughborough University for their kind hospitality.

\section{REFERENCES}

1. S. I. Agafonov and E. V. Ferapontov Systems of conservation laws of Temple class, equations of associativity and linear congruences in P4, Manuscripta Math 106 (2001), 461488.

2. S. I. Agafonov and E. V. Ferapontov Systems of conservation laws in the setting of the projective theory of congruences: reducible and linearly degenerate systems, Diff. Geom. Appl. 17 (2002), 153-173.

3. S. I. Agafonov and E. V. Ferapontov, Systems of conservation laws from the point of view of the projective theory of congruences, Izv. RAN, ser. mat. 60 (1996), No. 6, 3-30.

4. S. I. Agafonov and E. V. Ferapontov, Theory of congruences and systems of conservation laws, J. Math. Sci. 94 (1999), No. 5, 1748-1792.

5. B. Dubrovin, Geometry of 2D topological field theories, in Integrable systems and quantum groups, Lecture Notes in Mathematics, No. 1620 (Springer-Verlag, 1996), 120-348.

6. B. A. Dubrovin and S. P. Novikov, Hydrodynamics of weakly deformed soliton lattices. Differential geometry and Hamiltonian theory, Uspekhi Mat. Nauk 44 (1989), No. 6, 29-98.

7. E. V. Ferapontov, Dupin hypersurfaces and integrable Hamiltonian systems of hydrodynamic type which do not possess Riemann invariants, Diff. Geom. Appl. 5 (1995), $121-152$.

8. E. V. Ferapontov, Isoparametric hypersurfaces in spheres, integrable nondiagonalizable systems of hydrodynamic type, and $N$-wave systems, Diff. Geom. Appl. 5 (1995), 335-369.

9. S. P. Finikov, Theory of congruences (M-L., Gostekhizdat, 1950).

10. A. Jeffrey, Quasilinear hyperbolic systems and waves, Research Notes in Math., No. 5, (Pitman, London, 1975).

11. P. O. Lax, Hyperbolic systems of conservation laws, Comm. Pure Appl. Math. 10 (1957), 537-566.

12. E. Mezzetti and D. Portelli, Threefolds in $P^{5}$ with a 3-dimensional family of plane curves, Manuscripta Math. 90 (1996), No. 3, 365-381.

13. P. J. Olver, Classical invariant theory (Cambridge University Press, 1999).

14. G. Ottaviani, On 3 -folds in $P^{5}$ which are scrolls, Ann. Scuola Norm. Sup. Pisa Cl. Sci. (4) 19 (1992), No. 3, 451-471.

15. F. Palatini, Sui sistemi lineari di complessi lineari di rette nello spazio a cinque dimensioni, Atti Ist. Veneto 60-2 (1900), 371-383.

16. B. Sévennec, Géométrie des systèmes hyperboliques de lois de conservation, Mémoire (nouvelle série), No. 56, Supplément au Bulletin de la Société Mathématique de France, 122 (1994), 1-125.

17. S. P. Tsarev, The geometry of Hamiltonian systems of hydrodynamic type. The generalized hodograph transform, Math. USSR Izv. 37 (1991), 397-419.

18. B. Temple, Systems of conservation laws with invariant submanifolds, Trans. Amer. Math. Soc. 280 (1983), No. 2, 781-795.

19. P. De Poi and E. Mezzetti, Linear congruences and hyperbolic systems of conservation laws, to appear. 